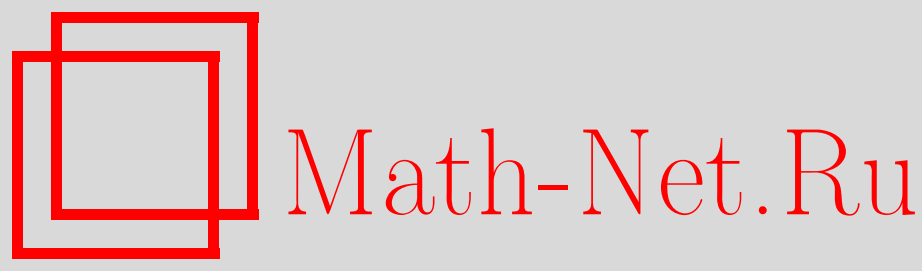

М. М. Степанова, К. С. Шефов, С. Ю. Славянов, Мультикритериальный алгоритм глобального поиска минимума в задаче оптимизации реактивного силового поля, ТМФ, 2016, том 187, номер 1, 177-194

DOI: https://doi.org/10.4213/tmf9159

Использование Общероссийского математического портала Math-Net.Ru подразумевает, что вы прочитали и согласны с пользовательским соглашением http://www . mathnet.ru/rus/agreement

Параметры загрузки:

IP : 18.209 .158 .208

26 апреля 2023 г., 13:58:09

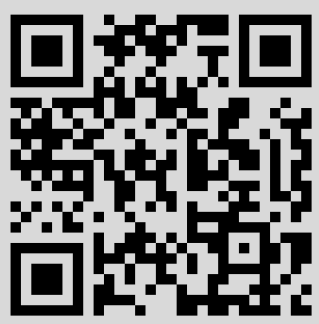




\title{
МУЛЬТИКРИТЕРИАЛЬНЫЙ АЛГОРИТМ ГЛОБАЛЬНОГО ПОИСКА МИНИМУМА В ЗАДАЧЕ ОПТИМИЗАЦИИ РЕАКТИВНОГО СИЛОВОГО ПОЛЯ
}

\begin{abstract}
Представлен новый мультикритериальный алгоритм глобального поиска минимума. Выполнена проверка его работоспособности на функциях Растригина и Михалевича. Обсуждается выбор целевой функции и дополнительных критериев поиска применительно к задаче оптимизации потенциала ReaxFF (Reactive Force Field). Рассматривается способ упорядочения всех параметров по степени их влияния на целевую функцию.
\end{abstract}

Ключевые слова: молекулярная динамика, потенциал взаимодействия, химически-реактивные системы, реактивное силовое поле, оптимизация параметров, поиск глобального экстремума.

DOI: $10.4213 / \operatorname{tmf9159}$

\section{1. ВВЕДЕНИЕ}

Квантовые расчеты в системах многих частиц с различными параметрами на основе дифференциальных уравнений крайне трудоемки и неэффективны. Классические молекулярно-динамические подходы, которые часто используются для крупномасштабных систем, как правило, опираются на статические связи и фиксированные парциальные заряды. Эти ограничивает их применимость только системами без химических реакций. В последние годы предложено несколько способов снять эти ограничения [1]-[4]. Наиболее гибким и перспективным представляется вариационный подход с реактивным силовым полем ReaxFF (Reactive Force Field) [5], [6], параметризация которого выполняется на основе точных квантовых расчетов для малых кластеров. Потенциал ReaxFF изначально разрабатывался с целью описать химическую реактивность, диссоциацию и формирование химических связей, дефекты, поверхностные эффекты и т. п., но затем он успешно применялся для описания самых разнообразных систем (см., например, работы [7]-[10]).

${ }^{*}$ Санкт-Петербургский государственный университет, Санкт-Петербург, Россия.

E-mail: mstep@mms.nw.ru, slav@ss2034.spb.edu

†ООО “Оракл девелопмент СПБ”, Санкт-Петербург, Россия. E-mail: k.s.shefov@gmail.com 
B настоящей статье объектом исследований является силовое поле ReaxFF, использующееся при моделировании химических реакций методами молекулярной динамики. Форма молекулярно-динамического потенциала ReaxFF подобна используемой во многих нереактивных силовых полях, где энергия системы есть сумма различных энергетических вкладов. Принципиальное отличие ReaxFF заключается в использовании не жесткого метода связывания, а метода порядка связей. Порядок связи характеризует кратность связи между конкретной парой атомов в соединении и выражается формулой

$$
B O_{i j}=\sum_{l} \exp \left\{p_{l 1}\left(\frac{r_{i j}}{r_{l}}\right)^{p_{l 2}}\right\},
$$

где индекс $l$ пробегает типы ковалентной связи и принимает значения $\sigma, \pi$ и $\pi \pi$; $r_{i j}$ - расстояние между атомами; $p_{l 1}, p_{l 2}, r_{l}$ - параметры. Порядки связей, которые при численном моделировании вычисляются для всех пар атомов и обновляются на каждом шаге по времени, позволяют описать образование и диссоциацию связей в ходе моделирования.

Общий вид потенциала таков:

$$
\begin{aligned}
& E_{\text {ReaxFF }}\left(\left\{r_{i j}\right\},\left\{r_{i j k}\right\},\left\{r_{i j k l}\right\},\left\{q_{i}\right\},\left\{B O_{i j}\right\}\right)= \\
&= E_{\text {bond }}+E_{\text {lp }}+E_{\text {over }}+E_{\text {under }}+E_{\text {val }}+E_{\text {pen }}+E_{\text {coa }}+ \\
&+E_{\text {tors }}+E_{\text {conj }}+E_{\text {hbond }}+E_{\text {vdWaals }}+E_{\text {Coulomb }} .
\end{aligned}
$$

Каждое слагаемое в правой части формулы отвечает за отдельный тип взаимодействия: ковалентное (слагаемые $E_{\text {bond }}, E_{\text {over }}, E_{\text {under }}$, характеризуется порядком химических связей), трехчастичное $\left(E_{\mathrm{val}}, E_{\mathrm{pen}}, E_{\text {соа }}\right.$, определяется валентными углами), четырехчастичное $\left(E_{\mathrm{tors}}, E_{\mathrm{conj}}\right.$, определяется двугранными валентными углами), кулоновское $\left(E_{\text {Coulomb }}\right)$, ван-дер-ваальсово $\left(E_{\mathrm{vdWaals}}\right)$, водородные связи $\left(E_{\mathrm{hbond}}\right)$ и энергия неподеленных электронных пар $\left(E_{\mathrm{lp}}\right)$. Полная энергия является суммой функций, зависящих от относительных положений атомов: положений пар $r_{i j}$, троек $r_{i j k}$ и четверок $r_{i j k l}$. Каждая из этих функций зависит от некоторых параметров, которые определяются выбором взаимодействующих химических элементов. Таким образом, весь потенциал является сложной многопараметрической функцией $E_{\mathrm{ReaxFF}}=f\left(p_{1}, \ldots, p_{N}\right)$; число параметров $N$ может быть более ста.

Подробный анализ всех слагаемых в формуле (1) позволяет сделать вывод, что $E_{\text {ReaxFF }}$ и его первая производная являются непрерывными функциями координат атомов даже в присутствии химических реакций. Чтобы получать адекватные результаты при моделировании химически взаимодействующих систем при помощи ReaxFF, необходимо подобрать параметры силового поля для конкретного класса моделируемых систем. Предварительно должна быть выбрана оптимизируемая целевая функция, зависящая от параметров потенциала ReaxFF и определяющая их оптимальность. Целевая функция представляет собой взвешенную сумму либо абсолютных значений, либо квадратов разностей неких характеристик химических соединений, входящих в конкретный класс. Характеристики сначала рассчитываются методами квантовой химии (или берутся из эксперимента), затем те же самые характеристики вычисляются с помощью ReaxFF и берутся разности первых и вторых. Набор характеристик, полученных методами квантовой химии или взятых из 
эксперимента, называется оптимизирующим набором. Соответствующие химические соединения определяют модели оптимизирующего набора.

Поиск глобального минимума целевой функции ввиду большой размерности области определения является весьма трудоемким. Один из вариантов решения этой задачи - использование алгоритма глобального поиска (АГП) [11], который дает гарантию сходимости на сетке. Заметим, что стандартные методы поиска минимума типа метода сопряженных градиентов, метода Пауэла или генетического метода в нашем случае плохо применимы, поскольку ищется глобальный, а не относительный минимум и целевая функция может характеризоваться большой овражностью [12].

Традиционно целевая функция, а именно взвешенная сумма квадратов отклонений, используется как единственная мера оптимальности. Такой способ имеет ряд недостатков. Во-первых, необходимо понять, как выбрать весовые коэффициенты. Во-вторых, даже при достаточно удачном выборе весов велик шанс, что при значительном снижении отклонений одних характеристик будет происходить одновременное увеличение отклонений других. Это может привести к неудовлетворительному описанию оптимизированным потенциалом ReaxFF конкретной группы химических соединений.

B нашей работе [13] представлен способ оптимизации параметров ReaxFF на основе усовершенствования АГП, который гарантированно сходится к глобальному минимуму в области определения целевой функции (сетке), прост в распараллеливании и позволяет быстро локализовать область расположения минимума функции. В настоящей работе мы предлагаем перестроить алгоритм так, чтобы в нем использовался не один, а несколько критериев оптимальности. Будем называть этот алгоритм мультикритериальным АГП, или МАГП. В работе обсуждается возможность распараллеливания МАГП, а также выполняется проверка его работоспособности на тестовых функциях Растригина и Михалевича. Предложенный подход связан с так называемым индексным АГП [14]. Определяющим в МАГП является выбор целевой функции и дополнительных критериев поиска применительно к конкретному потенциалу ReaxFF, а также упорядочение всех параметров по степени их влияния на целевую функцию.

\section{2. АЛГОРИТМ ГЛОБАЛЬНОГО ПОИСКА. БАЗОВЫЙ ВАРИАНТ}

АГП позволяет находить абсолютный минимум функции на сетке и основан на вероятностном подходе. На основе набора известных значений функции в точках сетки ищется интервал между соседними точками, на котором абсолютный минимум наиболее вероятен. На этом интервале берется точка, соответствующая математическому ожиданию положения минимума, вычисляется значение функции в ней (см. ниже формулу (4)). Точка добавляется в список известных значений, и происходит переход к следующей итерации. Алгоритм останавливается, когда расстояние между точками отрезка последовательных итераций становится меньше заданного значения.

Целевая функция $g(x)$ должна удовлетворять обобщенному условию Липшица на всем интервале поиска:

$$
\left|g\left(x_{1}\right)-g\left(x_{2}\right)\right| \leqslant K \rho\left(x_{1}, x_{2}\right)
$$


где $x_{1}$ и $x_{2}$ - любые числа из интервала поиска, $K$ - константа, а $\rho$ - метрика в пространстве $L_{1}, \rho\left(x_{1}, x_{2}\right)=f\left(\left|x_{1}-x_{2}\right|\right)$, причем функция $f$ имеет обратную, которую мы обозначим как $f^{-1}$.

Опишем шаги алгоритма [11]. Пусть $g(x)$ - целевая функция, определенная на сетке на отрезке $[a, b]$ вещественной оси. Обозначим через $z_{i}=g\left(x_{i}\right)$ значения целевой функции в точках $x_{i}$ отрезка $[a, b]$. Положим $x_{0}=a, x_{1}=b$ и вычислим $g(a)$ и $g(b)$. Пусть $x_{i}$ и $z_{i}$ для $i=0,1, \ldots, k$ нам уже известны, опишем $(k+1)$-ю итерацию.

1. Перенумеруем точки $x_{i}, i=0,1, \ldots, k$, в порядке возрастания.

2. Оценим максимальное абсолютное значение относительной первой разности:

$$
M=\max _{1 \leqslant i \leqslant k} \frac{\left|z_{i}-z_{i-1}\right|}{\rho_{i}},
$$

где $\rho_{i}=\rho\left(x_{i-1}, x_{i}\right), 0 \leqslant i \leqslant k$.

3. Положим

$$
m= \begin{cases}1, & M=0, \\ r M, & M>0,\end{cases}
$$

где $r>1$ есть заданный коэффициент надежности, зависящий от постоянной $K$ в условии (2).

4. Для каждого интервала $\left(x_{i-1}, x_{i}\right), 0 \leqslant i \leqslant k$, вычислим величину

$$
R(i)=m \rho_{i}+\frac{\left(z_{i}-z_{i-1}\right)^{2}}{m \rho_{i}}-2\left(z_{i}+z_{i-1}\right),
$$

которая называется характеристикой интервала. Она пропорциональна вероятности того, что глобальный минимум находится на этом интервале.

5. Определим интервал $\left(x_{t-1}, x_{t}\right)$, которому соответствует $R(t)=\max _{1 \leqslant i \leqslant k} R(i)$. Если максимум соответствует нескольким интервалам, то в качестве $t$ выбирается минимальное число.

6. Положим

$$
x_{k+1}=\frac{x_{t-1}+x_{t}}{2}-\frac{\operatorname{sgn}\left(z_{t}-z_{t-1}\right)}{2 r} f^{-1}\left(\frac{r\left|z_{t}-z_{t-1}\right|}{m}\right) .
$$

Если значение $x_{k+1}$ не принадлежит сетке, то оно заменяется ближайшим узлом сетки.

Алгоритм завершается, когда выполнено неравенство

$$
\rho_{t}<\varepsilon,
$$

где число $\varepsilon$ задано и определяет точность приближения к минимуму. Если $\varepsilon$ равно шагу сетки, то остановка вычислений происходит, когда точка (4), полученная на очередном шаге алгоритма, уже была получена на каком-либо из предыдущих шагов.

АГП гарантированно сходится к абсолютному минимуму функции на сетке, если на некоторой его итерации выполняется условие $m>4 K$ (см. соотношения (2) и (3)). Таким образом, условие сходимости задает конкретное значение параметра $r$ алгоритма. 


\section{3. МУЛЬТИКРИТЕРИАЛЬНЫЙ АЛГОРИТМ ГЛОБАЛЬНОГО ПОИСКА}

МАГП отличается от базового алгоритма тем, что работает не только с одной целевой функцией, но также учитывает некоторые дополнительные условия $g_{\nu}(x) \leqslant 0$, $1 \leqslant \nu \leqslant m$. Каждая функция $g_{\nu}(x)$ имеет ту же область определения, что и целевая функция, и в этой области удовлетворяет условию Липшица

$$
\left|g_{\nu}\left(x_{1}\right)-g_{\nu}\left(x_{2}\right)\right| \leqslant K_{\nu} \rho\left(x_{1}, x_{2}\right) .
$$

Опишем шаги мультикритериального алгоритма (см. также работу [14]).

Пусть $\phi(x)$ - целевая функция и $g_{k}(x), 1 \leqslant k \leqslant m,-$ функции-условия, заданные на сетке, принадлежащей отрезку $[a, b]$ вещественной оси. Определим числа $z_{i}$ следующим образом. Вычисляем значения $g_{k}\left(x_{i}\right)$, где $x_{i}$ - точка сетки, начиная с $k=1$. Если $g_{k}\left(x_{i}\right) \leqslant 0$, то проверяем следующее ограничение, увеличивая $k$ на единицу до тех пор, пока не будет выполнено неравенство $g_{k}\left(x_{i}\right)>0$. В итоге для данного $x_{i}$ получим число $\nu_{i}$, такое что $g_{k}\left(x_{i}\right) \leqslant 0$ при $1 \leqslant k<\nu_{i}$ и $g_{k}\left(x_{i}\right)>0$ при $k=\nu_{i}$. Как только найдено $k$, для которого $g_{k}\left(x_{i}\right)>0$, вычисления значений $g_{k}\left(x_{i}\right)$ прекращаются, число $z_{i}$ полагается равным $g_{\nu_{i}}\left(x_{i}\right)$. Если ни одно ограничение не нарушено, то $z_{i}=\phi\left(x_{i}\right)$, т. е. вычисляется значение целевой функции, а $\nu_{i}$ полагается равным $m+1$, и целевая функция $\phi(x) \equiv g_{m+1}(x)$. Набор $\left\{x_{i}, \nu_{i}, z_{i}\right\}$ называется испытанием в точке $x_{i}$, а $\nu_{i}$ - индексом этого испытания.

В начале алгоритма выберем произвольно любую точку сетки внутри интервала $(a, b)$ и вычислим $z_{i}$ и $\nu_{i}$ в этой точке. Пусть результаты первых $l$ испытаний известны.

1. Перенумеруем точки $x_{i}, i=1, \ldots, l$, в порядке возрастания. Положим $x_{0}=a$, $x_{l+1}=b$, а $z_{0}$ и $z_{l+1}$ будем считать неопределенными для удобства обозначений.

2. Проведем классификацию номеров $1 \leqslant i \leqslant l$ точек по числу ограничений задачи, выполняющихся в этих точках, путем построения множеств

$$
I_{\nu}=\left\{i: 1 \leqslant i \leqslant l, \nu=\nu\left(x_{i}\right)\right\}, \quad 1 \leqslant \nu \leqslant m+1,
$$

содержащих номера всех точек $x_{i}, 1 \leqslant i \leqslant l$, имеющих индексы, равные одному и тому же значению $\nu$. У граничных точек $x_{0}=a$ и $x_{l+1}=b$ индексы по определению равны нулю, и этим точкам сопоставляется множество $I_{0}=\{0, l+1\}$.

3. Построим множества

$$
S_{\nu}=I_{0} \cup \cdots \cup I_{\nu-1}, \quad 1 \leqslant \nu \leqslant m+1,
$$

содержащие номера всех точек из $\left\{x_{i}: 0 \leqslant i \leqslant l+1\right\}$, имеющих индексы меньше $\nu$, а также множества

$$
T_{\nu}=I_{\nu+1} \cup \cdots \cup I_{m+1} \cup I_{m+2}, \quad 1 \leqslant \nu \leqslant m+1,
$$

содержащие номера всех точек из $\left\{x_{i}: 0 \leqslant i \leqslant l+1\right\}$, имеющих индексы больше $\nu$ (по определению $I_{m+2}=\varnothing$ ).

4. Вычислим текущие нижние границы для неизвестных констант Липшица функций $g_{\nu}(x), 1 \leqslant \nu \leqslant m+1$ :

$$
\mu_{\nu}=\max \left\{\frac{\left|z_{i}-z_{j}\right|}{\rho_{i j}}: i, j \in I_{\nu}, i>j\right\},
$$


где $\rho_{i j}=\rho\left(x_{i}, x_{j}\right)$ - метрика из $(6)$. Если множество $I_{\nu}$ содержит менее двух элементов или если $\mu_{\nu}=0$ в (7), то принимаем $\mu_{\nu}=1$. Из выражения (7) следует, что значения $\mu_{\nu}$ являются неубывающими начиная с момента, когда в (7) рождается первое положительное значение $\mu_{\nu}$.

5. Для всех непустых множеств $I_{\nu}, 1 \leqslant \nu \leqslant m+1$, определим величины

$$
z_{\nu}^{*}= \begin{cases}\min \left\{z_{i}: i \in I_{\nu}\right\}, & T_{\nu}=\varnothing \\ -\varepsilon_{\nu}, & T_{\nu} \neq \varnothing\end{cases}
$$

где вектор $\varepsilon_{\mathrm{R}}=\left(\varepsilon_{1}, \ldots, \varepsilon_{m}\right)$ с неотрицательными компонентами является заданным и называется вектором резервов.

Пусть $\gamma \leqslant m$ таково, что $I_{\gamma} \neq \varnothing, T_{\gamma}=\varnothing$. Из (8) следует, что $z_{\gamma}^{*}>0$ и $z_{\nu}^{*} \leqslant 0$ для $1 \leqslant \nu<\gamma$. Тогда $\gamma$ следует рассматривать как индекс функции $g_{\gamma}(x)$, поиск минимума которой проводится на конкретной итерации. Отсюда $z_{\gamma}^{*}$ есть лучшая невозрастающая верхняя граница для минимума этой функции.

Вектор резервов, если он ненулевой, используется для ускорения сходимости алгоритма. При нулевом векторе $\varepsilon_{\mathrm{R}}$ точки испытаний, как правило, накапливаются не только в окрестности условного минимума целевой функции, но и в окрестностях недопустимых граничных точек областей

$$
Q_{i+1}=\left\{x \in Q_{i}: g_{i}(x) \leqslant 0\right\}, \quad 1 \leqslant i \leqslant m .
$$

При положительных компонентах вектора резервов этот эффект ослабляется, что видно из формул для характеристик $R(i)$ в шаге 6 алгоритма.

6. Для каждого интервала $\left(x_{i-1}, x_{i}\right), 1 \leqslant i \leqslant l+1$, вычислим характеристику

$$
R(i)= \begin{cases}\Delta_{i}+\frac{4\left(z_{i}-z_{i-1}\right)^{2}}{r^{2} \mu_{\nu}^{2} \Delta_{i}}-\frac{2\left(z_{i}+z_{i-1}-2 z_{\nu}^{*}\right)}{r \mu_{\nu}}, & i-1, i \in I_{\nu}, \\ 2 \Delta_{i}+\frac{4\left(z_{i}-z_{\nu}^{*}\right)}{r \mu_{\nu}}, & i-1 \in S_{\nu}, i \in I_{\nu}, \\ 2 \Delta_{i}+\frac{4\left(z_{i-1}-z_{\nu}^{*}\right)}{r \mu_{\nu}}, & i \in S_{\nu}, i-1 \in I_{\nu},\end{cases}
$$

где $\Delta_{i}=\rho\left(x_{i}, x_{i-1}\right)$. Величина $r>1$, как и выше, является параметром алгоритма и называется коэффициентом надежности.

7. Определим интервал $\left(x_{t-1}, x_{t}\right)$, которому соответствует максимальная характеристика $R(t)=\max _{1 \leqslant i \leqslant l+1} R(i)$. Если максимальная характеристика соответствует нескольким интервалам, то в качестве $t$ выбирается минимальное число. Вероятность нахождения минимума целевой функции на интервале $\left(x_{i-1}, x_{i}\right)$ пропорциональна $R(i)$.

8. Если индексы $\nu_{t}$ и $\nu_{t-1}$ концевых точек этого интервала различны, то мы выполняем очередное испытание в его серединной точке

$$
x_{l+1}=\frac{x_{t-1}+x_{t}}{2} .
$$

В противном случае, если индексы одинаковы, следует положить

$$
x_{l+1}=\frac{x_{t-1}+x_{t}}{2}-\frac{\operatorname{sgn}\left(z_{t}-z_{t-1}\right)}{2 r} f^{-1}\left(\frac{\left|z_{t}-z_{t-1}\right|}{\mu_{\nu}}\right) .
$$


Значение $x_{l+1}$ в формуле (11) имеет смысл математического ожидания минимума функции на том интервале, где на данный момент минимум расположен с максимальной вероятностью. Если значение $x_{l+1}$ не принадлежит сетке, то оно заменяется ближайшим ее узлом.

Условие завершения МАГП совпадает с условием (5) в базовом варианте.

МАГП сходится к глобальному минимуму функции на сетке, если на некоторой его итерации выполняются условия $r \mu_{\nu}>4 K_{\nu}$ для $1 \leqslant \nu \leqslant m+1$, где $K_{\nu}$ - константы из (6) для функций $g_{\nu}$, при этом $g_{m+1} \equiv \phi$ есть целевая функция.

Компоненты $\varepsilon_{\nu}$ вектора резервов следует выбирать так, чтобы были выполнены ограничения, налагаемые на них достаточными условиями сходимости МАГП. Так, значения $\varepsilon_{\nu}$, соответствующие активным в точке $x^{*}$ минимума целевой функции функциям-условиям $g_{\nu}(x)$ (т. е. выполняется равенство $\left.g_{\nu}\left(x^{*}\right)=0\right)$, должны удовлетворять неравенствам

$$
0 \leqslant 2 \varepsilon_{\nu}<K_{\nu}(\beta-\alpha)
$$

где $\beta-\alpha$ есть длина интервала $[\alpha, \beta] \subset Q_{m+1}$, который содержит точку минимума $x^{*}$. Если функции-условия $g_{\nu}(x)$, которым соответствуют $\varepsilon_{\nu}$, неактивны в точке минимума, т. е. $g_{\nu}\left(x^{*}\right)<0$, то $\varepsilon_{\nu}$ должны удовлетворять либо неравенствам (12), либо неравенствам

$$
0 \leqslant \varepsilon_{\nu}<\left|g_{\nu}\left(x^{*}\right)\right|
$$

Если точный вид функций-условий неизвестен, то векторы резервов можно вычислять методом адаптивных оценок [14]. Тогда компоненты вектора резервов будут выглядеть следующим образом:

$$
\varepsilon_{\nu}=\mu_{\nu} \varepsilon q, \quad 1 \leqslant \nu \leqslant m
$$

Здесь $\mu_{\nu}$ - числа, получающиеся на шаге 4 алгоритма, $\varepsilon$ - точность из условия остановки (5). Пусть $x^{*}$ - искомый минимум целевой функции, $x^{*} \in[\alpha, \beta]$, где $[\alpha, \beta]$ взяты из условия (12). Тогда $q \geqslant 1$ есть априорная оценка того, во сколько раз длина интервала $[\alpha, \beta]$ больше заданной точности $\varepsilon$.

Если нет возможности сделать какие-либо предположения о виде функций-условий, можно выполнять расчеты с нулевым вектором резервов. Мультикритериальный алгоритм при таком выборе и при выполнении остальных условий сходимости также будет работать, однако это заметно уменьшит скорость сходимости.

\section{4. ОБОБЩЕНИЕ АГП НА МНОГОМЕРНЫЙ СЛУЧАЙ}

Сеточная функция многих переменных приводится к сеточной функции одной переменной при помощи разверток, например кривой Пеано. В настоящей работе используется многомерный вариант АГП с неинъективной пеаноподобной разверткой. Будем считать без ограничения общности, что целевая функция и функцииусловия определены на сетке на гиперкубе. Если сетка задана на прямоугольном параллелепипеде, то его можно предварительно отобразить на гиперкуб. Развертка отображает сетку на гиперкубе в одномерную сетку. Неинъективность означает, что отображение, задаваемое такой разверткой, не взаимно однозначное, каждая точка сетки на гиперкубе размерности $N$ может иметь до $2^{N}$ прообразов на кривой. Иначе 


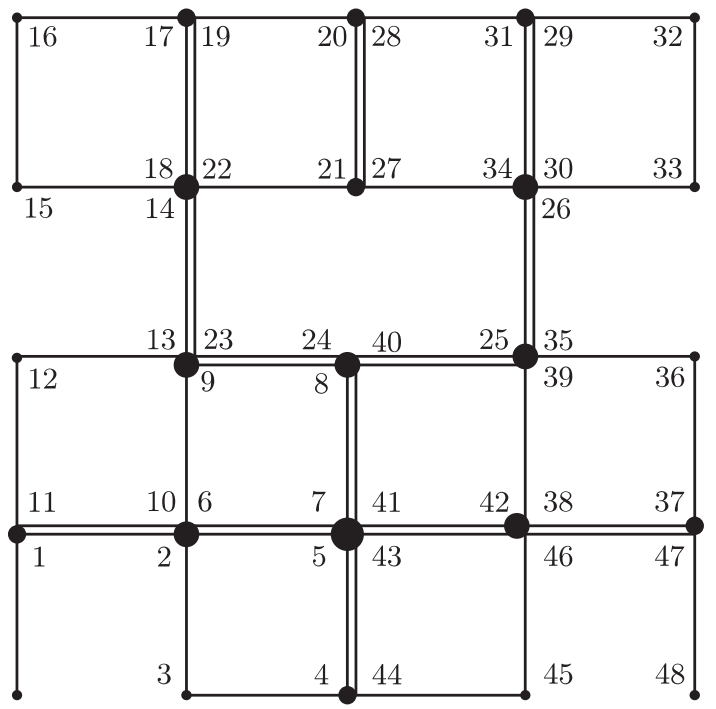

Рис. 1. Вид пеаноподобной кривой при $N=2$ и $m=3$.

говоря, кривая проходит через одни и те же точки гиперкуба несколько раз. Пеаноподобная развертка характеризуется плотностью $m$, которая определяет, сколько точек сетки приходится на каждое измерение гиперкуба, по формуле $2^{m}+1$.

Пример такой развертки с плотностью $m=3$ для области определения размерности $N=2$ показан на рис. 1 . Кривая на рис. 1 пробегает гиперкуб по точкам с возрастающими номерами. Размер точек на рис. 1 тем больше, чем большее число раз кривая проходит через них. Выбор именно такой развертки обусловлен тем, что при увеличении $m$ АГП можно продолжить с использованием уже имеющихся данных о значениях функции в точках, поскольку старые точки остаются на сетке при увеличении $m$.

Метрика $\rho$ из (2) при использовании развертки с пеаноподобной кривой имеет вид $\rho\left(x_{1}, x_{2}\right)=\sqrt[N]{\left|x_{1}-x_{2}\right|}$. Поиск минимума происходит на принадлежащей отрезку $[0,1]$ сетке, в которую отображается сетка на исходном гиперкубе размерности $N$. Полное число точек одномерной сетки определяется плотностью развертки $m$ и равно $2^{(m+1) N}-2^{m N}$. У всех точек одномерной сетки, имеющих одинаковые прообразы на многомерной сетке, значение целевой функции одно и то же. АГП, таким образом, дополняется вычислением прообразов.

\section{5. РАСПАРАЛЛЕЛИВАНИЕ МАГП}

Для распараллеливания МАГП используется метод вращающихся разверток [15]. Каждый параллельный процесс оперирует своей разверткой, повернутой по сравнению с основной на углы $\pm \pi / 2$ относительно какой-либо пары направлений. Всего можно сделать $N(N-1)$ таких поворотов для $N$-мерной области определения функции. Таким образом, всего алгоритм использует $N(N-1)+1$ процессов, каждый из которых выполняет МАГП и на каждой итерации сообщает свой результат всем 
остальным процессам. Параллельный алгоритм ускоряет сходимость и компенсирует последствия потери информации о близости точек гиперкуба при использовании развертки.

Нами был реализован параллельный многомерный МАГП на языке $\mathrm{C}++$ с использованием стандарта MPI для параллельной работы на системах с распределенной памятью. Для каждого параллельного процесса последовательность шагов одинаковая, процессы взаимодействуют друг с другом путем передачи сообщений.

Опишем алгоритм по шагам. Точки области определения функции (сетки на гиперкубе) обозначим через $Y_{i}$, значения целевой функции (или функций-условий) в этих точках обозначим как $Z_{i}$, значения индексов - как $V_{i}$, а точки сетки, принадлежащей отрезку $[0,1]$, на который отображается гиперкуб, обозначим через $X_{i}$.

Для инициализации алгоритма для каждого процесса вычислим $X_{1}$ - псевдослучайную точку сетки на отрезке $[0,1]$. Пусть мы уже имеем точки $X_{i}, 1 \leqslant i \leqslant l$, на отрезке $[0,1]$, а также значения $V_{i}$ и $Z_{i}, 1 \leqslant i \leqslant l-1$, из предыдущих $l-1$ итераций. Очередная итерация состоит из следующих шагов (для каждого процесса они одинаковые).

1. Сопоставим точке $X_{l}$ отрезка $[0,1]$ точку гиперкуба $Y_{l}$ при помощи отображения, обратного неинъективной развертке. Поскольку для каждого процесса развертки разные (повернутые), точки $Y_{l}$ в большинстве случаев различны для разных процессов.

2. Проведем МРІ-обмен точками $Y_{l}$ между всеми остальными процессами. После этого в каждом процессе точки $Y_{l}$ всех остальных процессов станут известными.

3. При совпадении точек $Y_{l}$ для разных процессов значение $Y_{l}$ для процесса с наименьшим порядковым номером остается неизменным, а для остальных процессов с теми же самыми $Y_{l}$ новые значения $Y_{l}$ получаются с помощью генератора псевдослучайных чисел. Это необходимо, чтобы не вычислять значения функции для одинаковых $Y_{l}$.

4. Для полученных $Y_{l}$ рассчитываем значения $V_{l}$ и $Z_{l}$. Этот шаг самый трудоемкий, поскольку подразумевает вычисление значений целевой функции и функцийусловий.

5. Проведем обмен значениями $Y_{l}, V_{l}$ и $Z_{l}$ со всеми остальными процессами. После этого для каждого процесса станут известными точки $Y_{l}$ всех остальных процессов и значения $V_{l}$ и $Z_{l}$ в них.

6. Из точки $Y_{l}$ получаем прообразы $X_{l, n_{l}}$. Число прообразов есть $n_{l} \leqslant 2^{N}$, где $N-$ размерность гиперкуба. Всем прообразам соответствуют одинаковые значения $V_{l}$ и $Z_{l}$.

7. Добавим тройки $\left\{X_{l, n_{l}}, V_{l}, Z_{l}\right\}$ в список. Список представляет собой одновременно связный список по всем точкам $X_{i}$, общее красно-черное дерево по точкам $X_{i}$, и отдельные красно-черные деревья для $X_{i}$ с одинаковыми $V_{i}$. Структура данных, хранящая тройки $\left\{X_{i, n_{i}}, V_{i}, Z_{i}\right\}, 1 \leqslant i \leqslant l$, состоит из ячеек, каждая из которых имеет поля $X$ и $Z$ двойной точности с плавающей точкой, целочисленное поле $V$, указатель на следующую ячейку однонаправленного связного списка, указатели на элементы-родители, левые и правые потомки как в общем красно-черном дереве, так и в частном красно-черном дереве по $V$ (всего 6 указателей), а также поля, хранящие цвета узла (черный или красный) в общем дереве и в частном дереве. Подобная структура данных делает быстрым поиск в ней по значению $X_{i}$ независимо от $V_{i}$. 
Поиск по значению $X_{i}$ при конкретном заданном $V_{i}$ также ускоряется, что полезно для шага 4 МАГП, сложность шага имеет порядок $O(\ln l)$. Пробег от начала до конца структуры по всем элементам имеет сложность $O(l)$ благодаря связному списку (что полезно для шага 6 МАГП). Узлы связного списка, в свою очередь, из-за наличия структуры красно-черного дерева будут всегда упорядочены по возрастанию значений $X_{i}$, что избавляет от необходимости проводить сортировку на шаге 1 .

8. Используя МАГП, описанный в разделе 3 , находим $X_{l+1}$. Заметим, что в памяти каждый раз сохраняются актуальные значения $\omega$ максимального индекса для всех проделанных испытаний, минимальное значение функции $g_{\omega}(x)$ и векторы значений $\mu_{\nu}, 1 \leqslant \nu \leqslant m+1$, вычисляемые на шаге 4 АГП. Дополнительное использование памяти значительно уменьшает время одного шага.

9. Проверяем, имеется ли $X_{l+1}$ в списке; если есть, ставим индикатор завершения $F_{p}, 1 \leqslant p \leqslant P$, в значение "истина"; здесь $P=(N-1) N+1$ есть число используемых процессов. Если значения $X_{l+1}$ нет в списке, но $\left|X_{l+1}-X_{l}\right|<\varepsilon^{N}$, где $\varepsilon$ - число из условия остановки $(5)$, то $F_{p}$ также принимает значение “истина". Далее производим обмен значениями $F_{p}$ с остальными процессами. Если $F_{p}$ для всех $1 \leqslant p \leqslant P$ находятся в значении "истина", то алгоритм останавливается, в противном случае происходит переход к следующей итерации.

Если алгоритм завершен, каждый процесс ищет в дереве $V_{\max }=\max _{1 \leqslant i \leqslant l} V_{i}$ значение $X_{\min }$ с минимальным $Z_{i}=Z_{\min }$ и находит соответствующую ему точку $Y_{\min }$. Пара $\left(Y_{\min }, Z_{\min }\right)$ есть результат работы алгоритма. У всех процессов набор пар $\left(Y_{l}, Z_{l}\right)$ один и тот же, следовательно, минимум тоже будет одинаковым. Таким образом, результатом работы алгоритма будет положение глобального минимума $Y_{\min }$ целевой функции с точностью до шага сетки либо с точностью $\varepsilon$ из условия (5). Значение глобального минимума будет равно $Z_{\min }$.

Усложнение параллельной реализации МАГП по сравнению с базовым вариантом не столь велико. Однако МАГП обладает преимуществом, поскольку в нем при поиске минимума учитывается несколько критериев. Например, если целевая функция представляет собой сумму неотрицательных величин, то МАГП позволяет потребовать, чтобы каждое из слагаемых не превышало некоторого заданного значения. Это преимущество является ключевым для задачи поиска параметров силового поля ReaxFF.

\section{6. ТЕСТИРОВАНИЕ МАГП НА ФУНКЦИЯХ РАСТРИГИНА И МИХАЛЕВИЧА}

Функция Михалевича имеет вид

$$
f(\mathbf{x})=-\sum_{i=1}^{d} \sin \left(x_{i}\right) \sin ^{2 p}\left(\frac{i x_{i}^{2}}{\pi}\right)
$$

Размерность $d=2$, параметр $p=10$, область определения есть $0 \leqslant x_{i} \leqslant \pi, i=1,2$. Такая функция имеет овражную структуру и два локальных минимума разной глубины. Для отображения в одномерную область использовалась неинъективная развертка плотности $m=12$ и 4097 точек сетки на одно измерение. Точность алгоритма выбиралась равной шагу сетки, $\varepsilon=0.000767$. 
Функция Растригина имеет вид

$$
f(\mathbf{x})=10 d+\sum_{i=1}^{d}\left[x_{i}^{2}-10 \cos \left(2 \pi x_{i}\right)\right] .
$$

Размерность $d=2$, область определения задавалась как $-5.12 \leqslant x_{i} \leqslant 5.12, i=1,2$. Функция обладает большим числом локальных минимумов, симметрично расположенных относительно начала координат, в котором находится абсолютный минимум функции. Плотность неинъективной развертки $m=12$. Точность алгоритма выбиралась равной шагу сетки, $\varepsilon=0.0025$.

Поскольку МАГП предполагает наличие хотя бы одной функции-условия, для функции Михалевича была введена функция

$$
g(\mathbf{x})=\left(x_{2}-2.8113\right)^{2}-0.5
$$

которая предопределяет сходимость АГП в точку $(2.2027,2.7113)$ менее глубокого минимума, поскольку более глубокий находится за пределами допустимой условием области.

Для функции Растригина использовалось два варианта условий, каждое из которых исключает самый глубокий минимум (начало координат). В первом случае применялась одна функция

$$
g(\mathbf{x})=-x_{2}+2.5
$$

которая должна обеспечить сходимость в точку минимума $(0,2.985)$. Во втором случае применялись две функции

$$
g_{1}(\mathbf{x})=-x_{2}+2.5, \quad g_{2}(\mathbf{x})=-x_{1}+1.5,
$$

для которых ожидалась сходимость в точку минимума $(1.99,2.985)$.

Исследовалась зависимость числа итераций, необходимых АГП для достижения заданной точности, от величины фиксированных компонент вектора резервов $\varepsilon_{\mathrm{R}}$. Значения всех компонент вектора $\varepsilon_{\mathrm{R}}$ выбирались равными друг другу. Во всех случаях параметр $r=4$.

На рис. 2а представлена зависимость числа итераций, выполненных МАГП до достижения условия остановки, от величины компонент $\varepsilon_{\nu}$ вектора резервов для функции Михалевича с функцией (15). На рис. 26 та же зависимость показана для функции Растригина с функцией (16) и функции Растригина с функциями-условиями (17). Штриховыми линиями на графиках отмечены значения компонент вектора резервов, при превышении которых нарушается условие (13): для всех трех случаев это $\varepsilon_{\nu}=0.49$, что следует из положения искомых минимумов и вида функцийусловий (15)-(17).

Как видно из рис. 2, увеличение значения компонент вектора резервов в пределах, допустимых условием (13), снижает число итераций в несколько раз. Однако при нарушении условия (13) дальнейшее увеличение значения $\varepsilon_{\nu}$ уже не снижает число итераций, и даже заметен небольшой рост этого числа. Таким образом, несмотря на то что в данном случае алгоритм сходится к ожидаемым значениям минимума при всех представленных векторах резервов, целесообразно выбирать их такими, чтобы они не нарушали условие (13). 


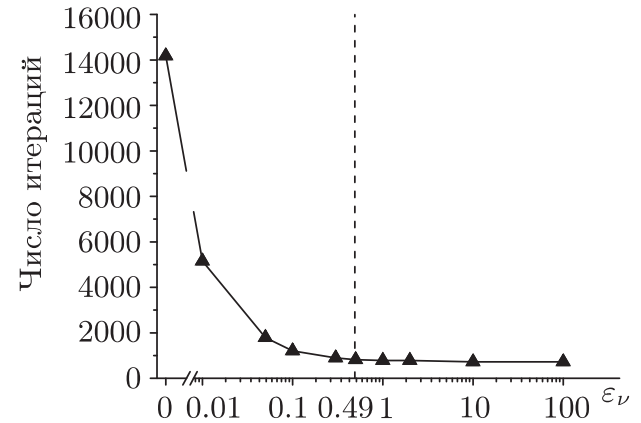

a

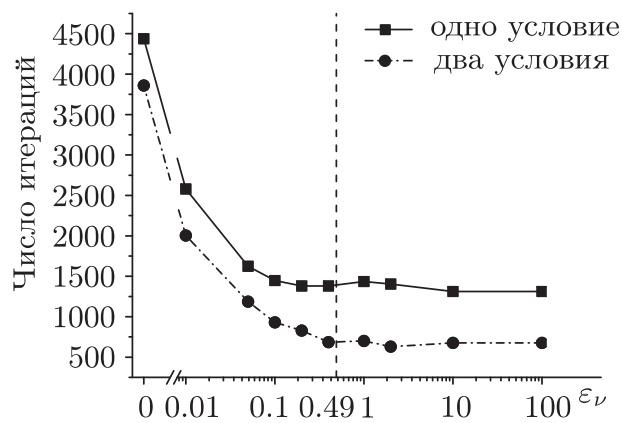

б

Рис. 2. Зависимости числа итераций от величины компонент вектора резервов для МАГП с функциями Михалевича (а) и Растригина (б).

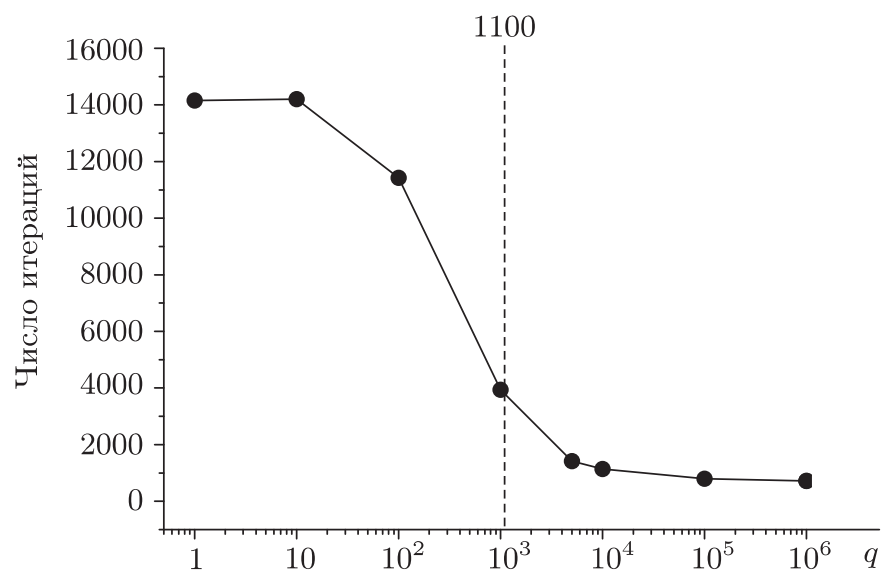

Рис. 3. Зависимость числа итераций от параметра $q$ для МАГП с функцией Михалевича.

Во многих случаях нельзя точно сказать, выполняется ли условие (13) для выбранных векторов резервов, поэтому мы предлагаем еще один способ выбора - адаптивный (см. раздел 3).

Нами проведено исследование зависимости числа итераций от параметра $q$ из соотношения (14) для адаптивного метода в случае функции Михалевича с условием, заданным функцией (15). Поскольку $q$ - априорная оценка того, во сколько раз длина интервала $[\alpha, \beta]$ больше заданной точности $\varepsilon$, можно оценить оптимальное значение $q$ на основании условия (15), числа точек сетки на параметр и вида кривой, порождаемой используемой разверткой. Условие (15) вырезает из области определения функции Михалевича полосу шириной примерно $14 \%$ от общей длины области. Плотность сетки равна 4097 точек на измерение. Отсюда следует, что длина интервала кривой, на котором лежит искомый минимум, по крайней мере в 1100 раз больше шага сетки, равного в данном случае точности. 
На рис. 3 показан график зависимости числа итераций МАГП от параметра $q$. Штриховой линией на графике отмечено значение $q=1100$, полученное выше. Из графика можно сделать вывод, что априорная оценка $q=1100$ в данном случае занижена и следует выбрать $q=10^{4}$ или $q=10^{5}$.

Созданная реализация МАГП адекватно работает на тестовых функциях. Максимальное увеличение вектора резервов в пределах ограничений (13) дает наилучшую скорость сходимости алгоритма.

\section{7. ПРИМЕНЕНИЕ МАГП ДЛЯ ПОИСКА ПАРАМЕТРОВ СИЛОВОГО ПОЛЯ RЕАХFF}

Общепринятый способ оптимизации параметров ReaxFF (1), в частности метод однопараметрического поиска [5], [16], предполагает выбор в качестве целевой функцию ошибки в виде

$$
\text { Error }=\sum_{i=1}^{n}\left[\frac{x_{i}^{\mathrm{QC} / \mathrm{Lit}}-x_{i}^{\mathrm{ReaxFF}}\left(p_{1}, \ldots, p_{N}\right)}{\sigma_{i}}\right]^{2} .
$$

Эта сумма представляет собой отклонение значения $x_{i}^{\mathrm{ReaxFF}}$ от $x_{i}^{\mathrm{QC} / \mathrm{Lit}}$, где $x_{i}^{\mathrm{QC} / \mathrm{Lit}}-$ данные оптимизирующего набора, a $x_{i}^{\mathrm{ReaxFF}}$ - микроскопические характеристики моделей оптимизирующего набора, рассчитанные с помощью методов молекулярной динамики с потенциалом ReaxFF (длины химических связей, валентные углы, эффективные заряды атомов, энергии атомизации и теплоты образования моделей, зависимости энергии кристаллов, приходящейся на одну ячейку, от объема ячейки). Числа $x_{i}^{\text {ReaxFF }}$ зависят от параметров потенциала $p_{1}, \ldots, p_{N}$. Весовые коэффициенты $\sigma_{i}$ имеют ту же размерность, что и $x_{i}$. Не существует какой-либо определенной техники выбора весов $\sigma_{i}$. Один из разумных способов был предложен создателями потенциала ReaxFF [5], согласно которому $\sigma_{i}$ выбираются исходя из ожидаемой разности квантовых расчетов и расчетов ReaxFF для $i$-й характеристики в конце оптимизации. Однако такой метод выбора является условным, всегда можно предложить иной.

Функцию вида (18) нельзя использовать как целевую для АГП, так как она не удовлетворяет условию Липшица (2), поскольку характеристики $x_{i}^{\text {ReaxFF }}$ берутся для моделей оптимизирующего набора, находящихся в состоянии оптимальной геометрии, т. е. сами являются результатами процедуры оптимизации поля ReaxFF по координатам атомов при фиксированных параметрах $p_{1}, \ldots, p_{N}$.

В нашей работе, чтобы удовлетворить условие Липшица, мы выбираем в качестве целевой другую функцию

$$
\text { Error }=\sum_{k=1}^{L} \sigma_{k}\left|U_{k}^{\mathrm{QC}}-U_{k}^{\mathrm{ReaxFF}}\right|+\sum_{k=1}^{L} \sigma_{L+k} \sqrt{\sum_{\alpha=1}^{A_{k}} \sum_{i=1}^{3}\left(F_{k \alpha i}^{\mathrm{QC}}-F_{k \alpha i}^{\mathrm{ReaxFF}}\right)^{2}} .
$$

Здесь $U_{k}$ - потенциальные энергии моделей оптимизирующего набора, $F_{k \alpha i}$ - компоненты сил, действующих на атомы каждой модели, $L$ - число моделей оптимизирующего набора, $A_{k}$ - число атомов в моделях, $\sigma_{k}$ - весовые множители. Индексы QC и ReaxFF означают, что характеристики были получены методами квантовой химии 
и молекулярной динамики с потенциалом ReaxFF. Функции $U_{k}$ зависят от параметров ReaxFF, т. е. $U_{k}^{\operatorname{ReaxFF}}=U_{k}^{\operatorname{ReaxFF}}\left(p_{1}, \ldots, p_{N}\right)$ и $F_{k \alpha i}^{\operatorname{ReaxFF}}=F_{k \alpha i}^{\operatorname{ReaxFF}}\left(p_{1}, \ldots, p_{N}\right)$. Как следует из явной формулы для ReaxFF [6], эта зависимость имеет непрерывные первые производные по оптимизируемым параметрам, что приводит к выполнению условий Липшица. Такой выбор является ключевым местом нашей работы.

При расчетах в рамках базового АГП [13] весовые множители $\sigma_{k}$ предлагается выбирать такими, чтобы средний вклад каждого слагаемого в (19) в целевую функцию был одинаковым. Среднее в данном случае рассчитывается по некоторому достаточно большому набору произвольных точек области определения функций $U_{k}^{\text {ReaxFF }}$ и $F_{k \alpha i}^{\text {ReaxFF }}$, т. е. точек пространства оптимизируемых параметров. Для вычисления средних вкладов сначала генерируется набор случайных векторов (порядка $10^{4}$ векторов). Далее для каждого вектора вычисляется значение каждого слагаемого в (19), при этом все $\sigma_{k}$ равны единице. Затем рассчитывается среднее значение $\theta_{k}$ каждого слагаемого по всем векторам и среднее значение $\Theta$ целевой функции. Новые веса $\sigma_{k}$ полагаются равными отношению $M / 2 L \theta_{k}$. Количество случайных векторов в наборе подчиняется требованию, что двукратное его увеличение не приводит к изменению $\sigma_{k}$ более чем на один процент.

Для вычисления целевой функции вида (19) оптимизация геометрии моделей не проводится. В отличие от функции (18), модели в функции (19) берутся статические, положения атомов в них всегда фиксированы. Это избавляет от необходимости проводить оптимизацию потенциала (1) по координатам атомов, однако вместо этого требуется достаточное количество статических конфигураций каждой модели оптимизирующего набора около ее положения равновесия, полученных методами квантовой химии.

В новом мультикритериальном алгоритме основной целевой функцией $\phi(x)$ также является функция (19). В качестве дополнительных критериев вводятся функцииусловия $g_{p}(x), 1 \leqslant p \leqslant m$, составленные из отдельных слагаемых сумм в правой части (19), а именно

$$
g_{p}(x)= \begin{cases}\sum_{i=1}^{L_{p}} \sigma_{i}\left|U_{i}^{\mathrm{QC}}-U_{i}^{\mathrm{ReaxFF}}\right|-\delta_{p}, & 1 \leqslant p \leqslant w, \\ \sum_{i=1}^{L_{p}} \sigma_{L_{p}+i} \sqrt{\sum_{\alpha=1}^{A_{i}} \sum_{j=1}^{3}\left(F_{i \alpha j}^{\mathrm{QC}}-F_{i \alpha j}^{\mathrm{ReaxFF}}\right)^{2}}-\delta_{p}, & w+1 \leqslant p \leqslant m,\end{cases}
$$

где $0 \leqslant w \leqslant m$, а $\delta_{p}-$ константа. Числа $\delta_{p}$ берутся из расчета средних значений сумм из (20), вычисленных на некотором количестве равномерно распределенных точек области поиска параметров ReaxFF. Наилучший набор таких точек можно получить при помощи ЛП $\tau$-последовательности (см. книгу [17] и раздел 8 настоящей работы). Такое задание функций $g_{p}(x)$ обеспечивает контроль за тем, что в ходе оптимизации группы слагаемых целевой функции не превышают заданных пределов.

При выполнении суммирования в (20) учитываются вклады оптимальных геометрий конкретной молекулы или кристалла (модели) для возможных деформаций вдоль выделенной степени свободы, например определенной химической связи, валентного угла или объема кристаллической ячейки. 


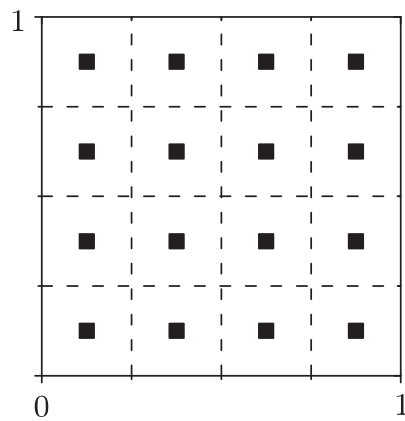

a

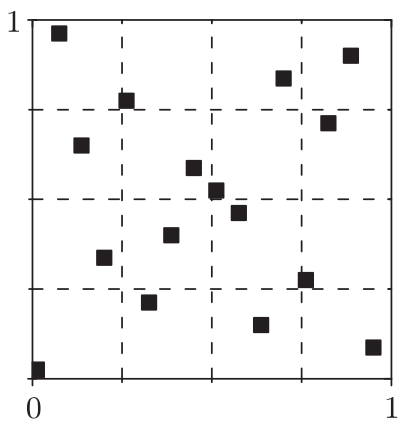

б

Рис. 4. Простая кубическая сетка из 16 точек (а) и сетка, построенная при помощи ЛП $\tau$-последовательности (б).

За счет наличия дополнительных критериев все веса $\sigma_{k}$ в формулах (19) и (20) полагаются равными единице. Векторы резервов выбираются методом адаптивных оценок (см. раздел 3 и пример в разделе 6).

Для вычисления $U_{k}^{\mathrm{ReaxFF}}$ и $F_{k \alpha i}^{\mathrm{ReaxFF}}$ из $(19)$ необходимо иметь реализацию самого силового поля ReaxFF. B настоящей работе используется молекулярно-динамический пакет LAMMPS [18].

\section{8. УПОРЯДОЧЕНИЕ ПАРАМЕТРОВ ЦЕЛЕВОЙ ФУНКЦИИ}

Недостатком АГП является экспоненциальный рост ресурсоемкости алгоритма с ростом числа одновременно оптимизируемых параметров ReaxFF. Это вынуждает отказаться от одновременного поиска сразу по всем возможным параметрам. Возникает необходимость упорядочения параметров по степени их важности. В качестве критерия важности берется величина коэффициента корреляции изменения слагаемых целевой функции (19) и изменения параметров ReaxFF.

Введем понятие вектора параметров, под которым будем понимать вектор, каждая компонента которого есть один из нескольких десятков изменяемых параметров ReaxFF. Выборку векторов параметров можно производить случайным образом, но это не гарантирует равномерного покрытия всей области определения целевой функции. Альтернативой является использование так называемых ЛП $\tau$-последовательностей [17]. Эти строго упорядоченные последовательности, построение которых основано на многократном двоичном делении области определения по всем измерениям, позволяют строить сетку на гиперкубе так, что точки этой сетки заполняют его как можно более равномерно.

Приведем пример сетки в единичном квадрате, полученной из 16 точек данной последовательности, и сравним эту сетку с простой кубической сеткой (см. рис. 4). Видно, что точки простой кубической сетки позволяют вычислить значение функции, определенной в квадрате, только при четырех различных значениях каждой из двух переменных. При этом сетка, построенная на основе ЛП $\tau$-последовательности, позволяет получить значение функции при 16 различных значениях каждой переменной для того же общего числа точек. Таким образом, ЛП $\tau$-последовательность 


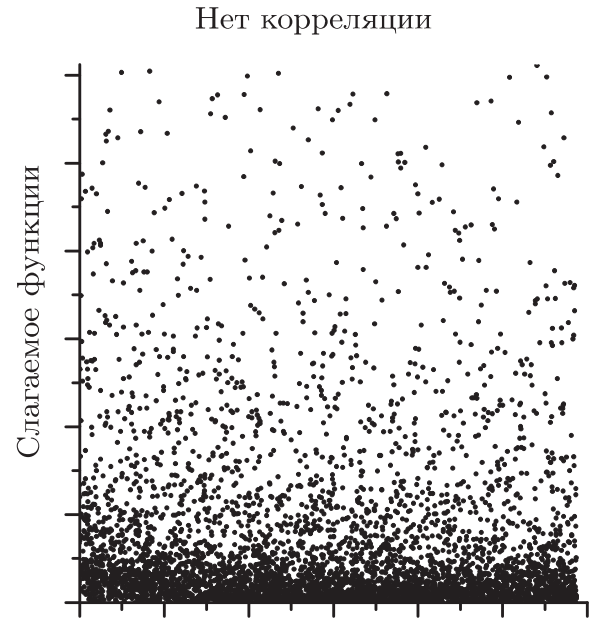

Параметр

a
Сильная корреляция

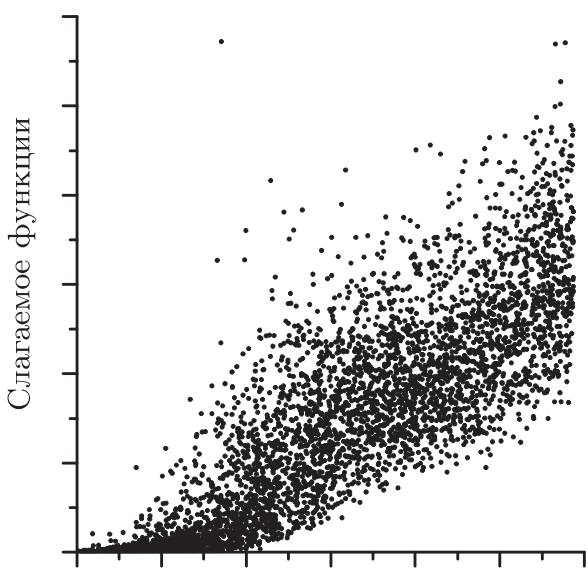

Параметр

б

Рис. 5. Примеры зависимостей значений слагаемых целевой функции от значения конкретных параметров при отсутствии корреляции (а) и в случае сильной корреляции (б).

позволяет выбрать более выгодное расположение точек в рассматриваемой области. Эти последовательности дают более равномерное распределение точек области определения функции, чем простая кубическая сетка и даже чем случайная выборка (согласно определению равномерности в книге [17]). Поэтому мы применяем ЛП $\tau$ последовательности.

В результате работы предложенного алгоритма параметры выстраиваются по убыванию максимальной величины корреляции изменения конкретного параметра и изменения конкретного слагаемого в формуле целевой функции. На рис. 5 изображены примеры зависимостей значений слагаемых целевой функции от конкретных параметров при выборке векторов параметров на основе ЛП $\tau$-последовательности при отсутствии корреляции (рис. 5а) и в случае сильной корреляции (рис. 5б).

Для запуска алгоритма необходимо установить границы изменения параметров. Схема алгоритма следующая.

1. Получаем при помощи генератора ЛП $\tau$-последовательностей достаточно большое число векторов, компоненты которых суть значения выбранных параметров в пределах заданных интервалов, в результате имеем матрицу $A$ размера $N \times R$, где $N$ - число параметров, а $R$ - число выборок.

2. Для каждой строки матрицы $A$ вычисляем значения слагаемых функции (19), которые записываем в строки матрицы $B$ размера $2 L \times R$, где $L$ - число моделей.

3. Нормируем матрицы $A$ и $B$ следующим образом:

$$
a_{i j}^{\text {norm }}=\frac{a_{i j}-\bar{a}_{i}}{\sqrt{R} \sigma_{i}}, \quad b_{i j}^{\text {norm }}=\frac{b_{i j}-\bar{b}_{i}}{\sqrt{R} \sigma_{i}} .
$$


Здесь $\bar{a}_{i}$ - среднее по столбцу матрицы, $\sigma_{i}$ - дисперсия по столбцу. Таким образом, мы получаем векторы изменений параметров относительно среднего и векторы изменений слагаемых функции (19) относительно среднего.

4. Вычисляем матрицу $C=A^{\mathrm{T}} B$ корреляций изменений параметров и изменений слагаемых целевой функции. Размер матрицы $C$ есть $2 L \times N$. Элементы $C_{i j}-$ коэффициенты корреляции.

5. Упорядочиваем строки матрицы $C$ по убыванию значения наибольшего по модулю элемента в строке. Каждая строка соответствует одному параметру.

Таким образом, в результате работы алгоритма параметры будут отсортированы по убыванию абсолютного значения коэффициента корреляции со слагаемыми целевой функции. Изменения параметров из начала списка будут влиять на целевую функцию сильнее, чем изменения параметров, следующих за ними. Это означает, что для оптимизации можно взять сначала несколько первых в списке параметров, затем несколько параметров, следующих за ними, и т. д. При необходимости проход по списку можно повторить.

\section{9. ЗАКЛЮЧЕНИЕ}

В работе представлен алгоритм оптимизации параметров молекулярно-динамического потенциала ReaxFF, основанный на мультикритериальном алгоритме глобального поиска и допускающий возможность задания и учета произвольного количества дополнительных условий. Введен специальный вид целевой функции, удовлетворяющей условию Липшица. Такая целевая функция позволяет существенно снизить временные затраты на каждом шаге численных вычислений. Также разработан и реализован алгоритм упорядочения параметров потенциала по степени влияния на целевую функцию на основе корреляции. Сравнение нового мультикритериального алгоритма с базовым вариантом показывает существенное преимущество первого.

Вычисления проводились на кластере кафедры вычислительной физики СПбГУ.

\section{Список литературы}

[1] D. W. Brenner, Phys. Rev. B, 42:15 (1990), 9458-9471.

[2] D. W. Brenner, O. A. Shenderova, J. A. Harrison, S. J. Stuart, N. Boris, S. B. Sinnott, J. Phys.: Condens. Matter, 14:4 (2002), 783-802.

[3] T. A. Halgren, W. Damm, Current Opinion Struct. Biol., 11:2 (2001), 236-242.

[4] S. J. Stuart, A. B. Tutein, J. A. Harrison, J. Chem. Phys., 112:14 (2000), 6472-6486.

[5] A. C. T. van Duin, S. Dasgupta, F. Lorant, W. A. Goddard III, J. Phys. Chem. A, 105:41 (2001), 9396-9409.

[6] K. Nomura, R. K. Kalia, A. Nakano, P. Vashishta, Comput. Phys. Commun., 178:2 (2008), 73-87.

[7] J. G. O. Ojwang, R. Van Santen, G. J. Kramer, A. C. T. van Duin, W. A. Goddard III, J. Phys. Chem., 128:16 (2008), 164714, 9 pp.

[8] D. Raymand, A. C. T. van Duin, D. Spångberg, W. A. Goddard III, K. Hermansson, Surface Sci., 604:9-10 (2010), 741-752.

[9] J. D. Deetz, R. Faller, J. Phys. Chem. B, 118:37 (2014), 10966-10978.

[10] S.G. Srinivasan, A. C. T. van Duin, P. Ganesh, J. Phys. Chem. A, 119:4 (2015), 571-580.

[11] Р. Г. Стронгин, Численные методы в многоэкстремальных задачах (информационностатистические алгоритмы), Наука, М., 1978.

7 Теоретическая и математическая физика, т. 187, № 1, 2016 г. 
[12] W. H. Press, S. A. Teukolsky, W. T. Vetterling, B. P. Flannery, Numerical Recipes in $C++$. The Art of Scientific Computing, Cambridge Univ. Press, Cambridge, 2002.

[13] К.С. Шефов, М.М. Степанова, Компъютерные исследования и моделирование, 7:3 (2015), 745-752.

[14] Р. Г. Стронгин, К. А. Баркалов, "О сходимости индексного алгоритма в задачах условной оптимизации с $\varepsilon$-резервированными решениями", Математические вопросы кибернетики, Сборник статей, 8, ред. С.В.Яблонский, Наука, М., 1999, 273-288.

[15] Р. Г. Стронгин, В.П. Гергель, К.А. Баркалов, Изв. вузов. Приборостроение, 52:10 (2009), 25-33.

[16] К. С. Шефов, М. М. Степанова, Программные продукты и системы, 2014, №2(106), $141-145$

[17] И. М. Соболь, Р. Б. Статников, Выбор оптималъных параметров в задачах со многими критериями, Дрофа, М., 2006.

[18] LAMMPS Molecular Dynamics Simulator, http://lammps.sandia.gov/.

Поступила в редакцию 1.02.2016 